\title{
THERMAL CONDUCTION IN METALS: MENTAL REPRESENTATIONS IN 5-6 YEARS OLD CHILDREN'S THINKING
}

\author{
George Kaliampos ${ }^{1}$, Konstantinos Ravanis ${ }^{* 2}$ \\ ${ }^{1}$ University of Nicosia, Cyprus \\ ${ }^{2}$ Department of Educational Sciences and Early Childhood Education, University of Patras, Greece \\ *Correspondence address: ravanis@ upatras.gr
}

Received: January $24^{\text {th }}, 2019$. Accepted: April 15 $5^{\text {th }}, 2019$. Published: April $29^{\text {th }}, 2019$

\begin{abstract}
In this paper, we present the findings of research on mental representations of thermal conduction on the metal of children aged 5-6 years. The research was carried out using interviews of 87 urban area kindergarten students in Greece. The children were asked for predictions and macroscopic descriptions of phenomena illustrated in 3 different tasks during which simple thermal conduction experiments were performed with both daily and non-daily materials. The research results clearly show the vast majority of children express mental representations which are far from the scientific models used in education. Moreover, many children can make predictions on heat conduction without being able to analyze their way of thinking. Finally, differences were found in heat conduction on familiar and non-familiar materials used by children. Findings of the current research study are likely to equip teachers with the appropriate basic tools for designing their teaching interventions in the lessons of heat and thermal conduction for children aged 5-6 years.
\end{abstract}

(C) 2019 Physics Education, UIN Raden Intan Lampung, Indonesia

Keywords: five-six years old students, mental representations, thermal conduction

\section{INTRODUCTION}

One of the main research topics within science education is the study of mental representations on concepts and phenomena of sciences (Borgerding \& Raven, 2018; Kaltakci-Gurel, Eryilmaz, \& McDermott, 2016; Métioui \& Trudel, 2012; Nasser, El Khouzai, \& Taoufik, 2018; Ravanis \& Boilevin, 2009). Recording and categorization of these representations enable the researcher to determine the problems of students' thinking and design the teaching interventions which lead to the construction of cognitive models which are compatible with those of Sciences (Delegkos \& Koliopoulos, 2018; Métioui \& Trudel, 2010; Ntalakoura \& Ravanis, 2014; Okulu \& OğuzÜnver, 2018; Sabirova \& Deryagin, 2018). A small part of this research is oriented in child thinking which falls into early childhood education, i.e. ages 4-8.
In this context, using different research methods and teaching practices, has been proven possible for children of 5-7 years to systematically approach the natural world and construct pre-concepts compatible with those of physics science in several cognitive learning areas such as optics (Delserieys, Impedovo, Fragkiadaki, \& Kampeza, 2017; Pantidos, Herakleioti, \& Chachlioutaki, 2017), astronomy and meteorology (Fragkiadaki \& Ravanis, 2015; Küçüközer \& Bostan, 2010; Papandreou \& Terzi, 2011; Saçkes, 2015), electric and magnetism (Christidou, Kazela, Kakana, \& Valakosta, 2009; Kada \& Ravanis, 2016; Kalogiannakis, Nirgianaki, \& Papadakis, 2018). In modern academic bibliography, these entities that are formed in children's mind from an early age are called precursor models (Delserieys, Jégou, Boilevin, \& Ravanis, 2018; Weil-Barais, 2001). 'Precursor models are cognitive entities 
which: a) interpose themselves between the original children's representations and the scientific models used in education, and b) allow pupils to put forth thought processes which, on the one hand, surmount the obstacles of their thinking and, on the other, are compatible with scientific ones. The structure and content of the precursor model are put together by a systematic matching of young children's difficulties and the relevant characteristics of the scientific reference model' (Ravanis, 2017).

Over the past 30 years, a number of researches have been geared towards studying the understanding of thermal phenomena by young children. Both Russell, Harlen \& Watt and Bar studied mental representations of evaporations for 5-11 years old children (Bar, 1989; Russell, Harlen, \& Watt, 1989). Bar and Galili, Levins as well as Ravanis and Bagakis designed and implemented specific teaching interventions for the transformation of children representations of 3-6 years on evaporation (Bar \& Galili, 1994; Levins, 1992; Ravanis \& Bagakis, 1998). Tytler also studied the development of mental representations of children of 67 years on evaporation and condensation (R Tytler, 2000). Ravanis, Papandreou, Kampeza and Vellopoulou used a series of experimental activities in order to create a precursor model for thermal expansion and contraction of metals in children's minds of 5-6 years (Ravanis, Papandreou, Kampeza, \& Vellopoulou, 2013). Another study recorded the mental representations of children aged 5-6 years on melting and solidification of salt that happens to remain always in a solid state in everyday life (Ravanis, 2014). Kampeza, Vellopoulou, Fragkiadaki, and Ravanis studied preschoolers' representations of the expansion thermometer, as well as their ability to distinguish it as a specialized technical tool (Kampeza, Vellopoulou, Fragkiadaki, \& Ravanis, 2016). Finally, in Cruz-Guzmán, García-Carmona and
Criado study with 2-4 year olds, an attempt was made to understand simple state changes by using everyday materials (Cruz-Guzmán, García-Carmona, \& Criado, 2017), while in Smith \& Samarakoon study, changes in the water cycle were revisioned through the usage of children's art and creativity aged 5 to 6 years old (Smith \& Samarakoon, 2017).

The analysis of both qualitative and quantitative data of these researches showed that the thought of small children has a 'local' nature and is mainly dominated by untreated daily experiences and prelogical forms of thinking (Bar \& Galili, 1994). Those representations expressed by children have their basis on certain aspects of the phenomena, materials, and devices they use (Ravanis \& Bagakis, 1998). Thus, for example, they connect the temperature of the objects to their size and attribute the thermal properties to the materials from which the objects are made (Russell Tytler, 2000). On issues regarding change of matter, young children believe that the continuous heating of water will finally make it inflate and spill (Ravanis, 2014). While they express the view that steam is created by boiling water, they are reluctant to take the point that steam becomes water again as it disappears or penetrates into solid objects. It should be noted that the issues of change of matter in which young children seem to respond satisfactorily are linked exclusively to those that occur on daily life phenomena and cannot be generalized in other materials.

A key notion which plays a major role in how an individual makes sense of thermal phenomena is that of thermal conduction. Undoubtedly, thermal conduction is a complex concept which can be explained in Natural Sciences in the basis of a molecular model of matter structure. However, for young children, we do not seek to process abstract models in their thinking. Instead, our goal is to enable them to approach conduction on a basis 
that the thermal entity propagates across the whole mass of metallic materials and is not restricted to the point of contact with the heat source. In this perspective, our aim in the current research was to record and present mental representations of children 5-6 years on thermal conduction.

\section{METHOD}

The sample of the survey was 87 children 5-6 years recruited from 5 classes of an urban nursery school in Greece. The survey involved all the children who agreed to 'play with us' and for which special permission was requested by their parents. The research was carried out through individual semi-guided interviews lasting approximately 15 minutes. None of the children had come across in his/her classroom to any kind of activity or reference concerning thermal conduction. The interviews were carried out by a researcher and were analyzed autonomously by two researchers (the degree of consensus between the two researchers was 91\%). A qualitative conversational micro-analysis (Hazel \& Bolden, 2013) of the data sets collected during the interview dialogues between the subject and the researcher was made. Data analysis was based upon the audio recorded dialogues and individual observation protocols. During the interviews, protocols of nonverbal observations were followed by the second researcher. The interviews were recorded and the data was analyzed through transcripts. In addition, specific protocols for observing non-verbal behaviors were kept for each child.

The following materials were used in the research: heat source of cotton and alcohol, fine copper tubes, glasses of water and wooden and metal spoons.

Children were prompt to involve in the 3 following tasks:

1. In the first task, we showed children two copper tubes $30 \mathrm{~cm}$ long and $1 \mathrm{~cm}$ in diameter. The ends of one tube were closed while the others were open. We asked the children to predict whether one end of the tube would heat up when the other was heated to the flame of cotton (Task 1a for the open tube, Task $1 \mathrm{~b}$ for the closed tube). For every answer, we kindly asked children to explain to us their way of thinking. With this task, we attempt to capture the mental representations of children in a situation in which they have no familiarity, as copper tubes are completely neutral and unknown objects to them.

2. In the second task, the experimental procedure was quite similar with two distinct differences though. The first one was that while the tubes were closed at both ends, their length differs as one was $10 \mathrm{~cm}$ long and the other 30 $\mathrm{cm}$ long. The second difference was that now we were asking for justifications on which tube would warm up first. With this second task, we try to investigate whether children understand that there is an entity that spread along the metal pipes.

3. In the third task, we asked children to predict whether a metal (Task 3a) or a wooden spoon (Task $3 b$ ) would warm up after immersing a portion of it in a glass of hot water. With this final task, we try to investigate how children deal with thermal conduction when they come across everyday objects they are familiar with. Our special goal is to note whether the children are able to distinguish heat conduction on metal from other material objects.

\section{RESULTS AND DISCUSSION Results}

During the interviews, the children were asked to predict the evolution of phenomena and to justify their predictions. From the discussions that emerged, the researcher tried to grasp the innermost thinking of students and record any kind of mental representation that was formed. 


\section{Task 1}

From the data analysis, three distinct answer categories emerged. In these categories were not included student's answers who pointed out that 'I do not understand the task' or 'I do not know the answer'.

Sufficient answers. In this type of responses, children predict that the two tubes will be heated along their entire length and justify their prediction by stating that gradually heating will finally occupy the entire tube. To quote a child response 'It will heat up the whole pipe .... it will slowly heat up everywhere .... (task 1a)', 'the same.... the heat will slowly go up to the end of the pipe (Task 1b)' (Subject 33).

Intermediate answers. In this type of answers, children predict that the end of the tubes will warm up, but either their justifications are based on reasoning that is inconsistent with the scientific one or they do not offer any kind of justification at all. Hence a student response'It will warm up.... the heat will pass through the pipe from both above and below.... (taskla)', 'It will warm up to the edge.... air will transfer the heat... (Task 1b)' (Subject 67).

Insufficient answers. In this type of responses, children do not seem to recognize that the end of the tubes will be finally heated up. For example, 'It will not warm up... the heat will not reach the other end... it's far away from the flame (Task1a)', 'It will not warm up now either...the heat will not go down there ... (Task 1b)' (Subject 12).

In the following table 1 we present the allocation of children responses in the above-mentioned categories

Table 1. The children's answers to Task 1: categories and frequencies

\begin{tabular}{llccc}
\hline No & \multicolumn{2}{c}{ Categories of answers } & Frequency & Percentage \\
\hline \multirow{2}{*}{1} & \multirow{2}{*}{ Sufficient } & Task 1a & 2 & $2.3 \%$ \\
& & Task 1b & 2 & $2.3 \%$ \\
2 & \multirow{2}{*}{ Intermediate } & Task 1a & 29 & $33.4 \%$ \\
& & Task 1b & 25 & $28.7 \%$ \\
\multirow{2}{*}{3} & \multirow{2}{*}{ Insufficient } & Task 1a & 34 & $39 \%$ \\
\multirow{2}{*}{4} & \multirow{2}{*}{ I don't know } & Task 1b & 38 & $43.7 \%$ \\
& & Task 1a & 22 & $25.3 \%$ \\
& & Task 1b & 22 & $25.3 \%$ \\
\hline & & & $\mathbf{8 7}$ & $\mathbf{1 0 0 \%}$
\end{tabular}

\section{Task 2}

In this second task, children responses were also divided into three distinct categories.

Sufficient answers. In this type of answers, children predict that the end of the smallest tube will heat up faster than the larger one. To justify their prediction children often referred to the spread of 'heat' into the metal part of tubes. To quote a child response '(heat) will first reach the end of the small pipe (Researcher: Could you explain to me why?). Because in the large pipe, heat has to walk through more metal in order to reach the edge' (Subject 72).

Intermediate answers. In this type of answers, children predict that the end of the smaller tube will warm up firstly, but either their justifications are based on reasoning that is inconsistent with the scientific one or they do not offer any kind of justification at all. For example, 'The smaller tube will warm up firstly (Researcher: Could you explain to me why?). Because it is the smaller one' (Subject 7).

Insufficient answers. In this type of answers, children do not seem to recognize 
that the end of the tubes will be finally heated up. Hence a student response 'The pipes will not heat up... just like the previous ones (Task1)...' (Subject 44).
In the following table 2 we present the allocation of children responses in the above-mentioned categories.

Table 2. The children's answers to the Task 2: categories and frequencies

\begin{tabular}{clcc}
\hline No & Categories & Frequency & Percentage \\
\hline 1 & Sufficient answers & 4 & $4.6 \%$ \\
2 & Intermediate answers & 21 & $24.2 \%$ \\
3 & Insufficient answers & 31 & $35.6 \%$ \\
4 & I don't know & 31 & $35.6 \%$ \\
\hline & Total & $\mathbf{8 7}$ & $\mathbf{1 0 0 \%}$ \\
\hline
\end{tabular}

\section{Task 3}

Finally, in the third task children's responses were also classified into three distinct categories.

Sufficient answers. In this type of answers, children predict that the metal spoon will warm up while the wooden spoon will not. However, as sufficient we categorize the answers of children who attribute this differentiation to the diverse nature of materials. To quote a child response 'the whole spoon will warm up... because... it is the same as the pots with spaghetti get warm up while cooking' (Task 3a)', this spoon is wooden...it will not warm up while being out of water (Task 3b)' (Subject 61).

Intermediateanswers.In this type of answers, children predict that the metal spoon will warm up while the wooden not.
Nevertheless, their answers are not related in any case with the nature of the material used. For example, 'the whole spoon will warm up (Researcher: Could you explain to me why?). As it is inside the hot water, it will normally warm up... (Task 3a)', I do not know... it will not warm up to the edge... hot water will not reach the end of the spoon... (Task 3b)' (Subject 71).

Insufficient answers. In this type of answers, children do not seem to take the view that metal spoon will warm up and wooden spoon will not. Hence a student response'It will not warm up... the heat does not come out of the hot water (Task 3a)', 'It can warm up and burn (Task 3b)' (Subject 4).

In the following table 3 we present the allocation of children responses in the above-mentioned categories.

Table 3. The children's answers to Task 3: categories and frequencies

\begin{tabular}{ccccc}
\hline No & \multicolumn{2}{c}{ Categories of answers } & Frequency & Percentage \\
\hline \multirow{2}{*}{1} & \multirow{2}{*}{ Sufficient } & Task 1a & 26 & $29.9 \%$ \\
& & Task 1b & 11 & $12.6 \%$ \\
2 & \multirow{2}{*}{ Intermediate } & Task 1a & 33 & $37.9 \%$ \\
& & Task 1b & 17 & $19.6 \%$ \\
\multirow{2}{*}{3} & \multirow{2}{*}{ Insufficient } & Task 1a & 24 & $27.6 \%$ \\
\multirow{2}{*}{4} & \multirow{2}{*}{ I don't know } & Task 1b & 42 & $48.3 \%$ \\
& & Task 1a & 4 & $4.6 \%$ \\
& & Task 1b & 17 & $19.5 \%$ \\
\hline & & & $\mathbf{8 7}$ & $\mathbf{1 0 0 \%}$ \\
\hline
\end{tabular}




\section{Discussion}

In the current research, we tried to investigate mental representations on thermal conduction of children aged between 5-6 years old. It seems that our findings are in line with other similar surveys in the field of Science Education. More specifically, mental representations are 'local' and stand opposite the scientific models that are used in formal education and are mainly influenced by everyday experiences.

Indeed, as it was clearly shown in the first task, only two children were able to describe the heat up of the tubes by using a mechanism of conduction. On the contrary, $1 / 3$ of children failed to address the phenomenon through conduction as they shared ideas such as 'heating via air'. What is worth mentioning here is that almost $1 / 4$ of children were unable to express any kind of prediction.

In the second task, we tried to examine whether children were able to approach heat as a natural entity that propagates on the tube and spends a distance at a certain time. This approach is common, as the literature suggests, for invisible, natural entities on which children recognize a relationship between time and distance (Boyes \& Stanistreet, 1991; Ravanis \& Kaliampos, 2018). Here, only four children were able to formulate spatiotemporal reasoning for the propagation of heat to the tubes. While almost $1 / 4$ of children predicted correctly that the end of the tubes will get heat up, they were unable to justify their answer in terms of heat conduction. What is more, from the remaining $70 \%$ of children, half did not make any correct prediction to the task while the rest did not give any kind of answer at all.

Our results in the third task explicitly showed that heat conduction is better understood when familiar objects are used. This happens even in the case that heat source is a warm amount of water instead of a flame, as $29.9 \%$ of the children explain in details how the heat will dissipate in the metal spoon while $12.6 \%$ predict that the phenomenon will not occur in the wooden spoon. What is more, several children give intermediate answers as $37.9 \%$ and $19.6 \%$ predict that the heat will be conducted in the metal but not in the wood, without being able thought to attribute this heat propagation to the nature of the material.

A special reference should be made to the intermediate type of answers given by children, as they appear in a high percentage among all three different tasks ranging from $19.6 \%$ to $37.9 \%$.In this kind of answers are both recorded the systematic ability of children to make a correct prediction as well as their inability to formulate descriptions compatible with thermal conduction.

Our research results explicitly show that mental representations of young children on thermal conduction stand opposite the scientific ones, whose formation constitute our ultimate goal in science education. These research data can be the basis for creating teaching activities in kindergarten

\section{CONCLUSION}

At this age, the representations of thermal conduction on metal seem to be strongly influenced by everyday life experiences such as the usage of domestic machines i.e. electric kitchen.

However, two of the above-mentioned findings, namely the high ranking of intermediate students' responses and the frequent students' references in the everyday world constitute the basis for creating learning situations that could favor the transition from intuitive mental representations to a precursor model for thermal conduction. The basic feature of such a model would be the ability to recognize the phenomenon of heat conduction across the mass of metal objects, a mental conquest that allows both appropriate descriptions and predictions. 
This expectation is consistent with the academic literature, as research on mental representations of thermal conduction of children aged 5-6 years has shown that it is possible to set precursor models with descriptive and predictive features for notions such as thermal expansion and contraction as well as changes of the water state.

This prospect for thermal conduction is very interesting, as it is a phenomenon on which a series of other similar thermal phenomena are based. The requirements for ongoing and dynamic interchanges, the mediating role of the teacher and the context correlation are factors for further research.

\section{REFERENCES}

Bar, V. (1989). Children's views about the water cycle. Science Education, 73(4), 481-500.

Bar, V., \& Galili, I. (1994). Stages of children's views about evaporation. International Journal of Science Education, 16(2), 157-174.

Borgerding, L. A., \& Raven, S. (2018). Children's ideas about fossils and foundational concepts related to fossils. Science Education, 102, 414439.

Boyes, E., \& Stanistreet, M. (1991). Development of pupils' ideas of hearing and seeing - the path of light and sound. Research in Science and Technology Education, 9, 223-244.

Christidou, V., Kazela, K., Kakana, D., \& Valakosta, M. (2009). Teaching magnetic attraction to preschool children: a comparison of different approaches. The International Journal of Learning, 16(2), 115-128.

Cruz-Guzmán, M., García-Carmona, A., \& Criado, A. M. (2017). Aprendiendo sobre los cambios de estado en educación infantil mediante secuencias de pregunta-predicción comprobación experimental. Enseñanza de Las Ciencias, 35(3), 175-193.

Delegkos, N., \& Koliopoulos, D. (2018). Constructing the "energy" concept and its social use by students of primary education in Greece. Research in Science Education. https://doi.org/doi: 10.1007/s11165018-9694-y

Delserieys, A., Impedovo, M., Fragkiadaki, G., \& Kampeza, M. (2017). Using drawings to explore preschool children's ideas about shadow formation. Review of Science, Mathematics and ICT Education, 11(1), 55-69.

Delserieys, A., Jégou, C., Boilevin, J. ., \& Ravanis, K. (2018). Precursor model and preschool science learning about shadows formation. Research in Science and Technological Education, 36(2), 147-164.

Fragkiadaki, G., \& Ravanis, K. (2015). Preschool children's mental representations of clouds. Journal of Baltic Science Education, 14(2), 267274.

Hazel, A., \& Bolden, G. (2013). The conversation analytic approach to transcription. In J. Sidnell\& T. Stivers. In The handbook of conversation analysis (56-76). Malden, USA: Wiley-Blackwell.

Kada, V., \& Ravanis, K. (2016). Creating a simple electric circuit with children between the ages of five and six. South African Journal of Education, 36(2), 1-9.

Kalogiannakis, M., Nirgianaki, G. ., \& Papadakis, S. (2018). Teaching magnetism to preschool children: the effectiveness of picture story reading. Early Childhood Education Journal, 46(5), 535-546. 
Kaltakci-Gurel, D., Eryilmaz, A., \& McDermott, L. C. (2016). Identifying pre-service physics teachers' misconceptions and conceptual difficulties about geometrical optics. European Journal of Physics, 37(4).

Kampeza, M., Vellopoulou, A., Fragkiadaki, G., \& Ravanis, K. (2016). The expansion thermometer in preschoolers' thinking. Journal of Baltic Science Education, 15(2), 185193.

Küçüközer, H., \& Bostan, A. (2010). Ideas of kindergarten students on the daynight cycles, the seasons, and the moon phases. Journal of Theory and Practice in Education, 6(2), 267-280.

Levins, L. (1992). Students' understanding of concepts related to evaporation. Research in Science Education, 22, 263-272.

Métioui, A., \& Trudel, L. (2010). Evolution of student teachers' conceptions about light following constructivist didactic activities. In Research in Didactics of the Sciences: Monograph (249-252). Krakov, Poland: Pedagogical University of Krakov.

Métioui, A., \& Trudel, L. (2012). The model of the rectilinear propagation of light and the study of the variation of the size of a shadow. US-China Education Review, 2(9), 173-186.

Nasser, N., El Khouzai, M., \& Taoufik, M. (2018). Analyses des représentations des apprenants de tronc commun Marocain en interactions mécaniques (3éme loi de Newton) - Cas de la direction provinciale de l'Éducation Nationale de Settat. European Scientific Journal, 14(36), 159-173.

Ntalakoura, V., \& Ravanis, K. (2014). Changing preschool children's representations of light: A scratch based teaching approach. Journal of
Baltic Science Education, 13(2), 191200.

Okulu, H. Z., \& OğuzÜnver, A. (2018). The process of facilitating knowledge acquisition and retention: An inquiry into magnetic poles with challenging questions. International Education Studies, 11(5), 25-37.

Pantidos, P., Herakleioti, E., \& Chachlioutaki, M. (2017). Reanalysing children's responses on shadow formation: a comparative approach to bodily expressions and verbal discourse. International Journal of Science Education, 39(18), 2508-2527.

Papandreou, M., \& Terzi, M. (2011). Exploring children's ideas about natural phenomena in kindergarten classes: designing and evaluating "eliciting activities." Review of Science, Mathematics and ICT Education, 5(2), 27-47.

Ravanis, K. (2014). Les représentations des enfants de 5-6 ans sur la fusion et la solidification du sel, comme support pour le déploiement des activités didactiques. International Journal of Research in Education Methodology, 6(3), 943-947.

Ravanis, K. (2017). Early Childhood Science Education: state of the art and perspectives. Journal of Baltic Science Education, 16(3), 284-288.

Ravanis, K., \& Bagakis, G. (1998). Science education in kindergarten: Sociocognitive perspective. International Journal of Early Years Education, 6(3), 315-327.

Ravanis, K., \& Boilevin, J. . (2009). A comparative approach to the representation of light for five-, eightand ten-year-old children: didactical perspectives. Journal of Baltic Science Education, 8(3), 182-190.

Ravanis, K., Papandreou, M., Kampeza, 
M., \& Vellopoulou, A. (2013). Teaching activities for the construction of a precursor model in 5-6 years old children's thinking: the case of thermal expansion and contraction of metals. European Early Childhood Education Research Journal, 21(4), 514-526.

Ravanis, K., \& Kaliampos, G. (2018). Mental representations of 14-15 years old students about the light propagation time. Jurnal Pendidikan Progresif, 8(2), 44-52.

Russell, T., Harlen, W., \& Watt, D. (1989). Children's ideas about evaporation. International Journal of Science Education, 11(5), 566-576.

Sabirova, F. M., \& Deryagin, A. V. (2018). The creation of junior schoolchildren's interest in the experimental study of physical phenomena using the elements of the technology of problem-based. International Journal of Engineering \& Technology, 7(2), 150-154.

Saçkes, M. (2015). Young children's ideas about earth and space science concepts. In Research in early childhood science education (35-36). Netherlands: Springer.

Smith, L. L., \& Samarakoon, D. (2017). Teaching kindergarten students about the water cycle through arts and invention. Journal of STEM Arts, Crafts, and Constructions, 2(1), 6078.

Tytler, R. (2000). A comparison of year 1 and year 6 students' conceptions of evaporation and condensation: dimensions of conceptual progression. International Journal of Science Education, 22(5), 447-467.

Tytler, R. (2000). A comparison of year 1 and year 6 students' conceptions of evaporation and condensation: dimensions of conceptual progression. International Journal of Science Education, 22(5), 447-467. https://doi.org/10.1080/09500690028 9723

Weil-Barais, A. (2001). Constructivist approaches and the teaching of science. Prospects, 31(2), 187-196. 